\title{
Smart Bins: Fallstudienbasierte Bewertung der Nutzenpotenziale von Füllstandssensoren in intelligenten Abfallbehältern
}

\author{
David Hoffmann • Ruben Franz $\mathbb{D} \cdot$ Florian Hawlitschek $・$ Nico Jahn
}

Eingegangen: 30. März 2021 / Angenommen: 5. August 2021 / Online publiziert: 6. September 2021

(C) Der/die Autor(en) 2021

Zusammenfassung Smart City-Strategien, gesellschaftliche Trends und übergeordnete Nachhaltigkeitsziele erfordern neuartige Ansätze in der Abfallentsorgung. Zentrales Element stellt dabei die Weiterentwicklung konventioneller Abfallbehälter zu intelligenten „Smart Bins“ dar. Hier verspricht insbesondere der Einsatz von Sensorik zur Erkennung von Füllgraden eine effizientere und effektivere Abhollogistik. Abfallbehälter werden üblicherweise in fest definierten Intervallen, unabhängig vom Füllgrad, geleert. Während eine verfrühte Abholung unnötige Aufwände und Emissionen zur Folge haben kann, kann eine zu späte Abholung in überfüllten Behältern und Littering-Verhalten resultieren. Zusätzlich kann der Einsatz von Sensorik in Abfallbehältern zur Verbesserung des Standplatzservice, einem besseren Behältermanagement und zu neuartigen digitalen Services beitragen. Der Einsatz von Sensorik erfordert jedoch ein Verständnis der technischen Rahmenbedingungen und den Möglichkeiten einer zielgerichteten Verwendung. Sensorik wird in der Branche noch nicht umfassend eingesetzt, auch die aktuelle Forschungslage ist fragmentiert und stützt sich überwiegend auf Simulationsstudien oder praktische Untersuchungen in laborähnlichen Szenarien. Dieser Artikel zeigt daher konkret die verschie-

\footnotetext{
David Hoffmann

Wirtschaftsbetriebe Duisburg - AöR, Duisburg, Deutschland

E-Mail: d.hoffmann@wb-duisburg.de

Ruben Franz $(\bowtie)$

Universität Duisburg-Essen, Essen, Deutschland

E-Mail: ruben.franz@uni-due.de

Florian Hawlitschek

FES Frankfurter Entsorgungs- und Service GmbH, Frankfurt, Deutschland

E-Mail: Florian.Hawlitschek@fes-frankfurt.de

Nico Jahn

AWISTA - Gesellschaft für Abfallwirtschaft und Stadtreinigung, Düsseldorf, Deutschland

E-Mail: NJahn@awista.de
} 
denen Ausprägungsformen einer sensorbasierten Messung von Behälterfüllgraden auf. Basierend auf einer multiplen Fallstudie mit drei Entsorgungsunternehmen werden anhand des praktischen Einsatzes von Sensoren zentrale Herausforderungen, Empfehlungen für weitere Pilotprojekte und Anforderungen an zukünftige Entwicklungen abgeleitet. Dieser Artikel bietet damit einerseits eine theoretisch und praktisch fundierte Entscheidungshilfe für Entsorgungsunternehmen, welche den Einsatz von Füllstandssensoren erwägen. Andererseits werden Wissenschaft und Praxis aufgerufen, die skizzierten Lösungsansätze für die identifizierten Herausforderungen mitzugestalten.

Schlüsselwörter Abfallentsorgung - Smart Bins · Füllstandsmessung · Sensorik · Internet-of-Things

\title{
Smart Bins: Case study-based benefit evaluation of filling level sensors in smart waste containers
}

\begin{abstract}
Smart city strategies, social trends and overriding sustainability goals call for novel approaches to waste management. The focal element here is the further development of conventional waste containers into intelligent "smart bins". Especially the use of sensor technology to detect filling levels in particular promises more efficient and effective waste logistics. Waste containers are usually emptied at fixed intervals, regardless of the filling level. While early waste collection may result in unnecessary expenses and emissions, late collection can result in overfilled containers and littering behavior. In addition, the use of sensor technology in waste containers may contribute to improved service levels, better container management, and novel digital services. However, the use of sensor technology requires an understanding of the technical framework and the possibilities of targeted use. Sensor technology is not yet used extensively in the industry, and the current research situation is also predominantly fragmented, relying mainly on simulation studies or practical investigations in laboratory-like scenarios. This article therefore specifically shows the different forms of sensor-based measurement of container filling levels. Based on a multiple case study with three waste management companies, challenges, recommendations for further pilot projects, and requirements for future developments are derived. To this end, this article provides a theoretically and practically sound decision-making aid for waste management companies considering the use of level sensors. Further, science and practice are invited to shape the solutions for the identified challenges.
\end{abstract}

Keywords Waste management - Smart bins · Filling level measurement · Sensor technology $\cdot$ Internet of Things

\section{Einleitung}

Im Rahmen der Sicherstellung des öffentlichen Gemeinwohls ist der wesentliche Auftrag der insgesamt 1676 in der deutschen Entsorgungswirtschaft tätigen Unter- 
nehmen (Destatis 2018) die Sammlung, Beförderung und Behandlung von Abfällen, also Stoffen, derer sich ihre Besitzer entledigen wollen oder müssen (Kranert 2017). Das gesamte Abfallaufkommen in Deutschland betrug im Jahr 2018 insgesamt 417,2 Mio. Tonnen und ist seit 2006 um fast 12\% gestiegen. Obwohl Deutschland dabei über eines der fortschrittlichsten Entsorgungssysteme mit der EU-weit höchsten Recyclingquote verfügt, wird die Entsorgungswirtschaft überwiegend als nicht-digitale Branche wahrgenommen (Wilts und Berg 2018). Digitale Technologien bieten jedoch vielfältige Potenziale, die gegenwärtigen und zukünftigen Herausforderungen der Entsorgungswirtschaft zu adressieren. Zum einen erhalten auch nicht originär digitale Konzepte wie Cradle-to-Cradle (McDonough und Braungart 2003), Sharing Economy (Hawlitschek et al. 2018) oder Zero Waste (Song et al. 2015) stetig mehr Aufmerksamkeit, die darauf abzielen, weniger Abfälle in die Stoffkreisläufe gelangen zu lassen (Zaman 2015) beziehungsweise die Einzelbestandteile von Produkten nach dem Ende von deren Nutzung möglichst vollständig dem Stoffkreislauf wieder zuzuführen. Zum anderen erfordern veränderte Erwartungshaltungen von Politik und BürgerInnen sowie zahlreiche Smart-City-Initiativen die Entwicklung von neuen intelligenten Applikationen, welche dabei helfen, die natürlichen Ressourcen zu schonen und die Nachhaltigkeit und Attraktivität von Städten zu steigern (D'Onofrio und Portmann 2017). Gängigen Smart-City-Zieldimensionen (z. B. Giffinger et al. 2007) und den Zielen für nachhaltige Entwicklung der Vereinten Nationen (United Nations 2015) folgend, sind daher auch Unternehmen der Entsorgungswirtschaft dazu aufgefordert, neue intelligente Wege für mehr Ressourcen-, Klima- und Umweltschutz und hohe Lebensqualität in Städten zu erkunden.

Einen Ansatzpunkt für digitale Innovationen im Bereich der Entsorgungswirtschaft bietet dabei die sensorbasierte Erfassung von Füllständen in Abfallbehältern für die Lösung kundenspezifischer Entsorgungsprobleme und daraus abgeleitete antizipative Anpassungen von Abfuhrintervallen und Anfahrtsrouten. Diese möglichen Nutzenpotenziale sind insbesondere im Hinblick auf Echtzeitsteuerung von Logistikprozessen sowie gesteigerten Konsumentenbedürfnisse nach ubiquitärer Verfügbarkeit von Informationen und smarten Produkt-Service-Bündeln relevant (Yoo et al. 2012; Hoffmann 2018). Bisher existieren aber nur wenige übergreifende, praktische Erfahrungsberichte und kaum Studien zu den Nutzenpotenzialen sensorgestützter Abfallsammelprozesse, sodass eine umfassende Beurteilung der möglichen Effizienzsteigerungen aber auch Kosten aussteht (Kamm et al. 2020; Hawlitschek 2021). Vor diesem Hintergrund präsentiert der vorliegende Beitrag Erkenntnisse zur sensorgestützten Füllstandsmessung aus explorativen Pilotprojekten bei drei Entsorgungsunternehmen. Basierend auf den empirischen Erkenntnissen werden die Nutzenpotenziale kritisch reflektiert, offene Herausforderungen aufgezeigt und sowohl Forschende als auch Unternehmen aus den Bereichen der Sensorik, Tourenplanung und Künstlicher Intelligenz (KI) zu deren Adressierung aufgerufen. Im folgenden Kapitel präsentieren wir zunächst eine allgemeine Definition von Smart Bins, die hypothetischen Nutzenpotenziale sowie die Ausprägungsformen von Füllstandsmessungen, welche als Grundlage für die explorative Untersuchung dienen. Im Anschluss daran stellen wir in Kapitel 3 die Vorgehensweise und die untersuchten Fallunternehmen vor. In Kapitel 4 präsentieren wir die Untersuchungsergebnisse und diskutieren diese. 
Ein zusammenfassendes Fazit und ein Ausblick schließen den Beitrag in Kapitel 5 $\mathrm{ab}$.

\section{Nutzenpotenziale von Smart Bins in der Entsorgungswirtschaft}

Als digitale Innovation der Entsorgungswirtschaft erfahren sogenannte Smart Bins zunehmend Aufmerksamkeit. Als Smart Bins werden Abfallbehälter bezeichnet, welche neuartige, durch digitale Technologien ermöglichte, Funktionen beinhalten und dadurch einerseits die Effizienz und Effektivität bestehender Prozesse der Entsorgungslogistik verbessern, aber auch völlig neue Prozesse und Services ermöglichen (Castro Lundin et al. 2017; Yerraboina et al. 2018; Kamm et al. 2020).

So können Smart Bins in ein Identifikations- und Wägesystem eingebunden werden, bei dem während des Kippvorgangs über RFID-Chips spezifische Behälterdaten, wie etwa Adresse und GebührenschuldnerIn, ausgelesen und die Leerung gewichtsabhängig abgerechnet wird (Kranert 2017). Zusätzlich ermöglichen elektronische Schlösser und Abfallschleusen ein spezifischeres Nutzungs- und Abrechnungsmanagement (VKU 2020) und damit perspektivisch auch völlig neue Gebühren- und Geschäftsmodelle. Zur Adressierung der Störstoffproblematik, welche die getrennte Erfassung von Wertstoffen stark beeinflusst, kann der Behälterinhalt mit Computer-Vision-Sensorik erfasst werden. Hierbei können einerseits Fehlwürfe und Störstoffe identifizieren werden, um diese vor der weiteren Verwertung zu entfernen. Andererseits können Wertstoffe in Restmüllbehältern identifiziert werden, um beispielsweise mittels Gamification-Ansätzen die Trennqualität zu steigern, etwa durch eine persönliche Nutzungsstatistik zum Trennverhalten in Relation zur Nachbarschaft (Höfle et al. 2019; Mittermayr und Klünsner 2020). Alle dabei erhobenen Datenpunkte können als umfassende Basis für darüber hinausgehende Szenarien verwendet werden, etwa zur Erstellung von ,Abfall-Heatmaps“, zum Training neuronaler Netze zur Abfallerkennung im öffentlichen Raum (Kang et al. 2019), oder zur Information von BürgerInnen oder zur Nachnutzung durch andere AkteurInnen in neuen Anwendungsfällen.

Neben den zuvor beschriebenen Einsatzszenarien von Smart Bins rückt in Wissenschaft und Praxis insbesondere der Einsatz von Sensoren zur Messung des Füllstands in Abfallbehältern und einer damit verbundenen Anpassung der Abfalllogistikprozesse in den Fokus (Castro Lundin et al. 2017; Kamm et al. 2020). Im Mittelpunkt der Betrachtung steht dabei die Erfassung der Füllstände von Abfallbehältern, die gegenwärtig in fest definierten Intervallen, unabhängig vom Füllgrad, entleert werden. Dies bezieht sich sowohl auf öffentliche Papierkörbe und Depotcontainer für Altglas, Altpapier oder Altkleider, als auch auf die gemeinschaftliche Sammlung von Abfall in konventionellen Müllgroßbehältern und Unterflursystemen. Neben dem unvermeidbar hohen Logistikaufwand durch die sehr hohe Anzahl an Behältern in einer Stadt können starre Abfuhrintervalle zu Ineffizienzen führen. So wurden beispielsweise in verschiedenen Kommunen je nach Fraktion (d.h. Abfallart) und Behältertyp mittlere Behälterfüllgrade von ca. 60-90\% (Sabrowski 2016), 60-85\% (Santjer 2018), 65-90\% (Stadt Halle 2014) und 55-80\% (Heller und Langheim 2018) beim Leerungszeitpunkt ermittelt. Die Abholung von zu gering befüllten Be- 
hältern ist wirtschaftlich ineffizient, verbraucht Energie, kostet Zeit und verursacht Emissionen. Abfallbehälter dürfen aber auch nicht zu spät abgefahren werden, um Überfüllungen, Verdichtungen und Beistellungen zu vermeiden. Ein Füllgrad von $80 \%$ sollte wegen saisonaler Schwankungen sowie geplanter oder ungeplanter Verschiebungen der Leerung nicht überschritten werden (Kranert 2017). Gerade im Hinblick auf die zunehmende Personalverknappung, steigende Energiepreise und einem allgemeinem Kostendruck könnte durch eine bedarfsorientierte Optimierung von Abfuhrintervallen und Anfahrtsrouten eine vermeintliche Reduktion von Arbeitsbelastung, Betriebsausgaben und Emissionen bei Erhöhung der Stadtsauberkeit erreicht werden.

Bisherige Studien zur sensorbasierten Optimierung von Abfalllogistikprozessen fokussieren sich jedoch überwiegend auf die Untersuchung unter laborartigen Bedingungen (Castro Lundin et al. 2017) oder sind rein hypothetischer Natur (Prasad und Kureethara 2018), so dass eine abschließende Bewertung der tatsächlichen Realisierbarkeit der zuvor genannten Nutzenpotenziale von Füllstandssensoren in

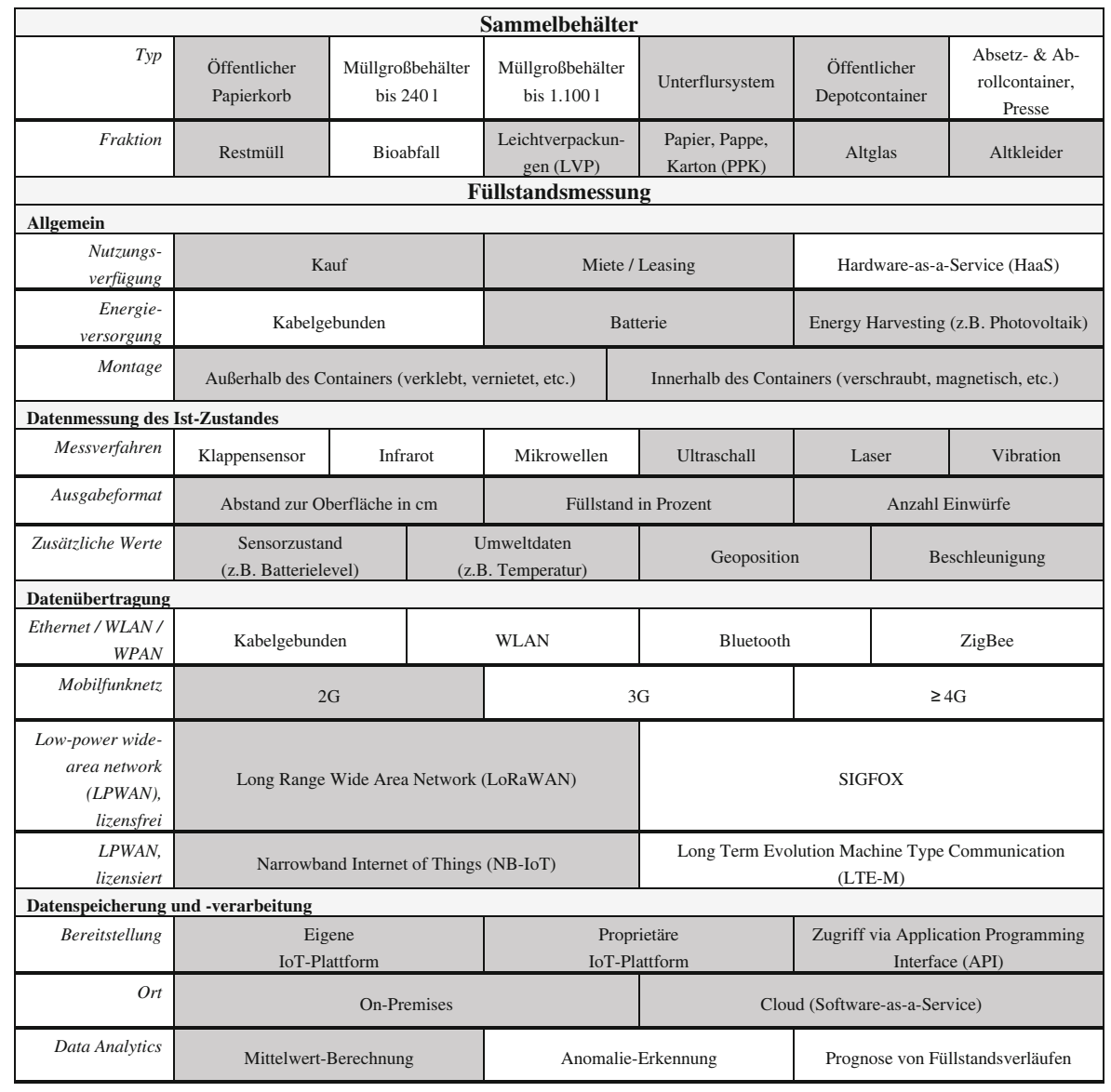

Abb. 1 Ausprägungen der Füllstandsmessungen in Smart Bins 
den Prozessen der Entsorgungswirtschaft noch weitestgehend unklar bleibt. Dieser Beitrag ordnet daher anhand des praktischen Einsatzes von am Markt verfügbaren Sensorlösungen in verschiedenen Kommunen den Reifegrad der Technologie vor dem Hintergrund der skizzierten Nutzenpotenziale ein. Die Ausprägungsformen der Füllstandsmessung in Abfallbehältern stellen wir in Abb. 1 dar. Diese Übersicht erlaubt es uns, das komplexe Konzept der Füllstandsmessung und die Beziehung zwischen den Merkmalen verständlich zu strukturieren und als Grundlage für die anschließende Analyse zu verwenden. Die Übersicht ergibt sich aus durchgeführten Marktrecherchen und den Piloteinsätzen der beteiligten Fallunternehmen. Innerhalb der Tabelle sind die Sensormerkmale markiert, die im Rahmen der im nächsten Kapitel beschriebenen Fallstudie ausgeprägt waren.

\section{Untersuchungsdesign und Vorstellung der Fallunternehmen}

Die Vorgehensweise des vorliegenden Beitrags folgt Empfehlungen für die explorative, aktions- und gestaltungsorientierte Erarbeitung von Problemlösungen mit mehreren Fallstudien in verteilten Innovationskontexten (Eisenhardt 1989; Sein et al. 2011). Primäres Ziel der Studie war die Erprobung von verschiedenen Sensortechnologien zur Füllstandsmessung in unterschiedlichen Abfallbehältern in mehreren Kommunen und der Vergleich der Ergebnisse. Es sollte ergründet werden, ob traditionelle Abfalllogistik-Prozesse durch den Einsatz von Füllstandssensorik perspektivisch anhand einer sensorbasierten, bedarfsgerechten Disposition effektiver und nachhaltiger gestaltet werden können. Damit soll insbesondere das Fehlen allgemeingültiger Handlungsempfehlungen und Erfahrungswerte zum Einsatz der Messtechnologie adressiert werden.

In drei Fallunternehmen der Entsorgungswirtschaft wurden unterschiedliche Ansätze bei der Untersuchung von Füllstandssensoren verfolgt. Vor dem Hintergrund der unternehmensspezifischen Aufgabengebiete, individueller Anforderungen und des geplanten Projektumfangs wurden je nach Fallunternehmen unterschiedliche Sensortypen und Behälter ausgewählt. Im Rahmen von Stakeholder-Interviews (z. B. mit FahrerInnen und DisponentInnen) wurden weitere Perspektiven zur Zwischenevaluationen berücksichtigt. Zusätzlich erfolgten Erfahrungsaustausche zwischen den Fallunternehmen. Um nach den fallindividuellen Auswertungen eine Übertragbarkeit der Resultate für andere Entsorgungsunternehmen zu ermöglichen, erfolgte im Anschluss eine Reflektion und Verallgemeinerung der Erkenntnisse. Tab. 1 fasst die Eigenschaften und Vorgehensweisen der Fallunternehmen zusammen.

\section{Fall 1 - AWISTA - Gesellschaft für Abfallwirtschaft und Stadtreinigung mbH}

Die Kontrolle und Reinigung ausgewählter, stark verunreinigter DepotcontainerStandplätze für Papier, Pappe und Karton (PPK) im öffentlichen Raum ohne Sensorik erfolgt durch ein tägliches Anfahren mit Straßenreinigungsteams. Zukünftig sollen Füllstandssensoren als automatisches Frühwarn- und Meldesystem fungieren, um Verunreinigungen der Standplätze entgegenzuwirken und diese möglichst adhoc zu beseitigen. Die Sensorik ermittelt einen definierten Zeitraum, in dem kein Einwurf mehr erfolgt ist, um bedarfsgerechter Reinigungs- und Kontrollfahrten zu 


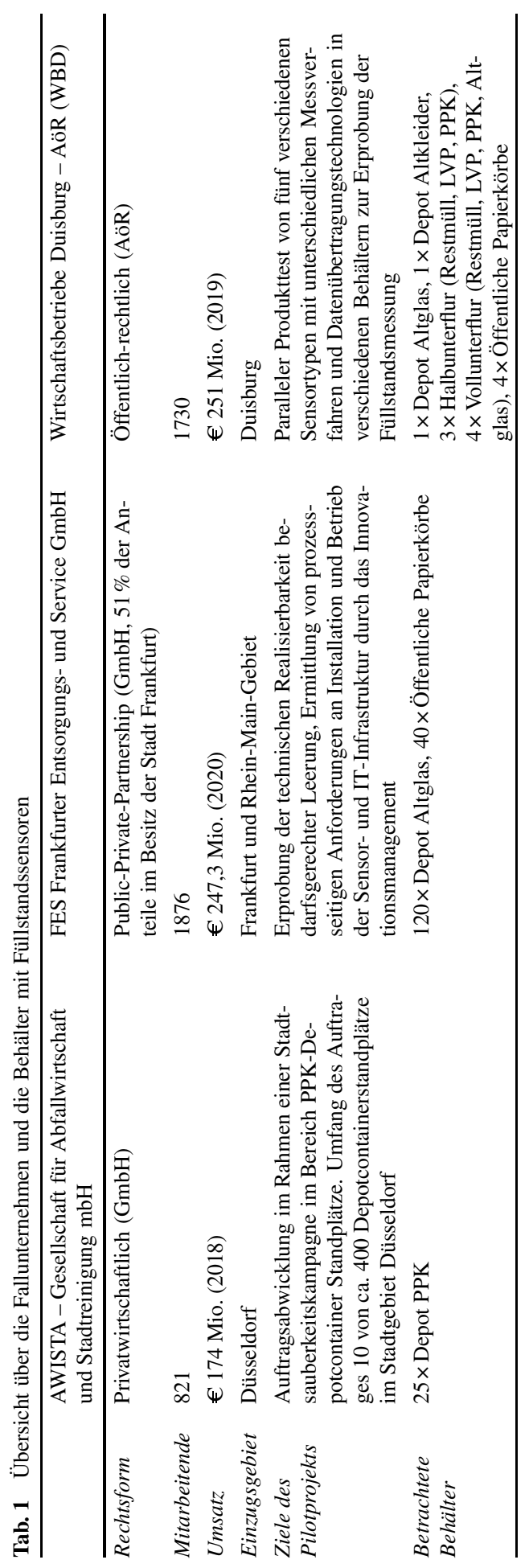


disponieren. Für das Pilotprojekt wurden 25 PPK-Depotcontainer mit einem Sensor versehen. Prämisse bei der Sensorauswahl war die leichte Montage, eine lange Nutzungsdauer, geringe projizierte Wartungsaufwände, eine hohe Robustheit sowie eine möglichst hohe Genauigkeit bei der Füllstandsmessung. Auf Basis der vorgenannten Anforderungen wurde ein wartungsfreier Sensor gewählt, der außen auf den Depotcontainern angebracht wird, die Energie über Photovoltaikzellen erzeugt und den Füllgrad anhand von historischen Messwerten und durch Einwürfe erzeugte Vibrationsmustern berechnet. Die Daten werden über Narrowband Internet of Things (NB-IoT) Funktechnologie übertragen.

Fall 2 - FES Frankfurter Entsorgungs- und Service GmbH (FES) Die FES erarbeitet im Rahmen des auf Nachhaltigkeit und Digitalisierung fokussierten Innovationsportfolios Potenziale und Einsatzmöglichkeiten einer bedarfsgerechten Entsorgung. Dabei geht es vor allem um die passgenaue Leerung von Abfallbehältnissen im öffentlichen Raum, welche zum einen Überfüllungszustände und die einhergehende Verschmutzung des Umfelds vermeiden, zum anderen aber die aus jeder Leerung resultierenden Emissionen von Lärm und Schadstoffen minimieren soll. In diesem Zusammenhang wurde ein Pilotbetrieb von batteriebetriebenen Ultraschallsensoren an 120 Depot-Altglascontainern und 40 innerstädtischen Papierkörben erprobt, um insbesondere die technische Realisierbarkeit eines flächendeckenden Einsatzes sowie die prozessseitigen Anforderungen an Installation und Betrieb der Sensor- und IT-Infrastruktur zu ermitteln. Die Ermittlung des Füllstandes via Ultraschall in Glascontainern wurde als defacto Marktstandard gegenüber anderen Verfahren präferiert und basierend auf den existierenden Erfahrungswerten auf den Anwendungsfall in Papierkörben übertragen. Aus Skalierbarkeits- und Kostengründen wurde ein eigens beauftragtes LoRaWAN-Netz zur Datenübertragung verwendet.

Fall 3 - Wirtschaftsbetriebe Duisburg - AöR (WBD) Die WBD haben in 13 unterschiedlichen Abfallbehältern parallel bis zu fünf Sensortypen eingesetzt mit dem Ziel, einen breiten Überblick über die Möglichkeiten und Grenzen der Technologie zu erhalten. Dazu haben die WBD drei verschiedene, batteriebetriebene UltraschallSensortypen $(2 \times 2$ G- $\& 1 \times$ LoRaWAN-Datenübertragung $)$ in einem Altglas-Depotcontainer, einem Altkleider-Depotcontainer, drei Halbunterflurbehältern (HUF) in einem Wohngebiet und vier Vollunterflurbehältern (VUF) in einem Gewerbegebiet installiert. Der LoRaWAN-Sensor war in der Lage, aus mehreren Messwerten zwischen zwei Datenübertragungen Mittelwerte zu berechnen. Zusätzlich wurden vier Sensoren des Typs, den die AWISTA ebenfalls verwendete, in einem Altglas-Depotcontainer, einem Altkleider-Depotcontainer, einem Halb- und einem Vollunterflurbehälter untersucht. Außerdem wurden in vier öffentlichen Papierkörben LoRaWANSensoren verbaut, welche die Füllgrade über Lasermessungen ermitteln. Für das Projekt wurden alle Sensoren auf eine halbstündige Messung und Datenübertragung konfiguriert.

Zum Abgleich von Messwerten mit den Ist-Füllständen wurden bei allen Fällen die Daten je Sensortyp über die proprietären Plattformen der Sensoranbieter abgerufen, wurden als tabellarischer Wochenbericht per Mail übermittelt, oder bei LoRaWAN-Sensoren direkt an eigene beziehungsweise städtische IoT-Plattformen 
übertragen. Während der Nutzungsphase erfolgten regelmäßige Abgleiche der Messwerte mit Daten, die anhand von manuellen Beobachtungen erhoben wurden.

\section{Ergebnisse}

\subsection{Ergebnisse zum praktischen Einsatz von Füllstandssensoren}

Fall 1 - AWISTA Ziel der Technologieerprobung bei der AWISTA war es, erste Erfahrungen zur Messtechnologie zu sammeln, um später über die Anbindung zu bestehenden Betriebsführungssystemen zu entscheiden. Auch erst in einer späteren Phase soll entschieden werden, ob die Technik auf alle PPK-Depotcontainerstandplätze ausgeweitet werden soll. Um den Füllstand für PPK-Depotcontainer zu bestimmen, müssen die besonderen Eigenschaften von PPK gegenüber anderen Wertstoffen berücksichtigt werden. PPK weist - im Gegensatz zum Beispiel zu Altglas keine kompaktierbaren Eigenschaften auf: Dies bedeutet, dass sich vor dem Einwurf zusammengefaltete Pappe nach dem Einwurf in den Container wieder entfalten kann und dadurch freies Behältervolumen ausfüllt. Aufgrund dieser Eigenschaft ist nicht jedes Messverfahren für die Messung des Füllstandes bei PPK-Depotcontainern geeignet. Hier bietet die Analyse der durch Einwürfe erzeugten Behälterschwingungen einen Vorteil gegenüber Verfahren, bei der die Zeit zwischen dem Aussenden eines Impulses und dem Eingang des Echos gemessen wird (wie z. B. bei Ultraschall).

Das Training der Algorithmen wurde in einem Zeitraum von vier Monaten (März-Juni 2020) durchgeführt. Im Kalibrierungszeitraum wurden rund 31.000 Einwürfe und 2700 Referenzfüllstände erfasst, letztlich konnte eine Genauigkeit der Füllstandsberechnungen von $94,5 \%$ erreicht werden. Zudem zeigte sich, dass die PPK-Depotcontainer am turnusmäßigen Leerungstag einen durchschnittlichen Füllgrad von $60 \%$ aufweisen. Die in Düsseldorf an Depotcontainerstandplätzen häufig beobachteten Verschmutzungen etwa durch Beistellungen wurden daher nicht auf tatsächliche Überfüllungen der Behälter, sondern auf das NutzerInnenverhalten und Faktoren wie die Sperrigkeit und Größe von Pappen und Kartonagen zurückgeführt.

Fall 2 - FES Erste Pilot- und Testsysteme mit Ultraschallsensorik wurden im Bereich der Altglascontainer und Papierkörbe erfolgreich installiert. Je nach Containermaterial kamen dabei entweder Schraub- oder Nietverfahren mit entsprechenden individuellen Vor- und Nachteilen bei Installation und Wartung zum Einsatz. Die Übertragung über die LoRaWAN-Infrastruktur verlief entsprechend der guten Netzabdeckung an den Pilot-Standorten insgesamt problemfrei. Die Ultraschall-Sensordaten legen eine brauchbare Genauigkeit im Bereich der Altglas-Depotcontainer nahe. Gleichzeitig ist die Genauigkeit bei Papierkörben allerdings zu gering, um aktuell einen flächendeckenden Einsatz zu rechtfertigen. Weiterhin kommt es mitunter zu Schwierigkeiten bei der Anpassung bestehender Abläufe (wie der Reparatur, dem Versetzen oder dem Austausch von Containern), sodass beispielsweise einzelne Standortinformationen manueller Nacharbeit bedürfen. Während also die Prozesse zur Installation und Wartung der Technik weitestgehend zuverlässig und beherrschbar erscheinen, stellt die Ableitung von Optimierungspotenzialen und vor 
allem die Umsetzung im Betrieb ein deutlich komplexeres Problem dar. In diesem Zusammenhang setzt FES auf Kooperationen mit ausgewiesenen Forschungspartnern, um mögliche Optimierungsansätze und -potenziale zunächst rechnerisch zu ermitteln, um sie im Folgeschritt in der Praxis zu erproben. Erste Analysen des Füllverhaltens ausgewählter Altglas-Depotcontainer zeigen, dass die durchschnittliche Befüllung einiger Container lediglich bei etwa $50 \%$ zum Zeitpunkt der Leerung liegt. Dies veranschaulicht die existierenden Optimierungspotentiale, die sich durch die Erhebung entsprechender Daten ergeben. Dennoch bedarf es zunächst weiterer Analysen, um auf Basis der erhobenen Daten für die betroffenen Fachbereiche klar kalkulierbare Optimierungsvorschläge und Prozessempfehlungen zu entwickeln. In Summe ist daher ein Technologietransfer vom Pilotbetrieb in die täglichen Arbeitsabläufe als mittelfristig vielversprechend, wenn auch nicht kurzfristig realisierbar einzuschätzen.

Fall 3 - WBD Über einen Zeitraum von sechs Wochen erfolgte jeweils an fünf Tagen pro Woche eine manuelle Erfassung der Füllgrade in den Behältern durch erfahrene Abfallkontrolleure. Bereits wenige Tage nach Beginn der Datenerhebung wurde festgestellt, dass keine ausreichende Verbindung zu den LoRaWAN-Sensoren in den drei HUF und im Altglas-VUF hergestellt werden konnte. Auch durch eine Verlegung zu anderen Behälterstandorten konnte keine Verbindung hergestellt werden. Dies wurde auf das in Duisburg noch im Aufbau befindliche LoRaWAN-Netzwerk und begrenzte Konfigurationsmöglichkeiten der Sensoren zurückgeführt. Daher wurde entschieden, diese vier Sensoren aus dem Test und der weiteren Analyse zu entfernen. Drei der vier Sensoren in den öffentlichen Papierkörben wurden im Testzeitraum trotz stabiler Befestigung wiederholt gestohlen, zudem fiel das LoRaWANGateway im Untersuchungszeitraum aus. Daher wurde entschieden, die Papierkörbe aus der Untersuchung zu entfernen. Einer der Ultraschallsensoren $(1 \times$ VUF Leichtverpackungen (LVP)) fiel aufgrund eines Elektronikdefekts im Testzeitraum aus und wurde nicht ersetzt. Die Zuverlässigkeit der LoRaWAN-Datenübertragung schwankte standortindividuell erheblich (40-88\% der erwarteten Datenpakete) und Übertragungsausfälle waren im zeitlichen Umfang von einer Stunde bis zu mehreren Tagen zu beobachten. Die Übertragung über NB-IoT und $2 \mathrm{G}$ erfolgte nahezu ohne Verluste.

Im Anschluss an den Testbetrieb wurden die Sensorwerte mit den manuellen Füllstandsprotokollen verglichen. Da die Algorithmen der Vibrationssensoren im Projektzeitraum nicht abschließend auf die Behälter kalibriert werden konnten, konnten zu diesen keine finalen Rückschlüsse zur Genauigkeit gezogen werden. Eine lineare Regressionsanalyse hat gezeigt, dass die Füllstandsverläufe aller Ultraschallsensoren - auch bei der nicht-kompaktierbaren Fraktion PPK - annähernd der Füllkurve entsprechen, welche durch das WBD-Personal dokumentiert wurden. Auch wenn die Gesamtheit der gemittelten Messwerte oft nahe an der Reallinie liegen, gab es jedoch häufig Ausreißer und hohe Standardabweichungen in den einzelnen Messwerten zu beobachten. Je nach Behältertyp und Abfallfraktion wurden zudem systematische Fehler identifiziert, die durch die Einbauposition bedingt waren: So zeigten beispielsweise zwei Ultraschallsensoren, die gegenüberliegend in einem HUF LVP verbaut waren, jeweils signifikante Abweichungen nach oben beziehungsweise unten vom realen Füllstandsverlauf. Dies ergab sich durch die jeweilige Entfernung der 
Sensoren zur für die Nutzenden einfacher zugängliche Einwurfklappe. Die durchschnittlichen Befüllungsgrade zur Leerung im Beobachtungszeitraum variierten je nach Behältertyp und Fraktion (33-100\%). In der Regel wiesen die meisten Behälter nur selten einen kritischen Füllstand von über $80 \%$ über mehrere Tage auf und füllten sich im Intervall gleichmäßig. Jedoch wurden mehrere ausgedehnte Perioden (>5 Tage) über dem kritischen Füllstand bei zwei HUF beobachtet. Bei dem öffentlichen VUF Altglasbehälter fanden zudem mehrere Leerungsvorgänge bei Füllständen von deutlich unter $50 \%$ statt, ebenso bei den öffentlichen Depotcontainern für Altglas und Altkleider. Bei diesen Behältern waren zudem weniger gleichmäßige, sondern sprunghafte Anstiege beim Befüllungsgrad zu beobachten.

\subsection{Lessons Learned}

Die Pilotprojekte haben gezeigt, dass der Einsatz von Füllstandssensoren nicht zu vernachlässigenden Aufwand erzeugt. Dieser wird insbesondere durch Planungs-, Installations-, Kalibrierungs- und Wartungsmaßnahmen verursacht. Es muss berücksichtigt werden, dass neben dem Sensoreinbau, auch in Abhängigkeit von Behältertyp und Fraktion, weitere Tätigkeiten für den Betrieb erforderlich sind. So sind in der Regel über einen gewissen Zeitraum mehrere Nachjustierung zur Identifikation der optimalen Einbauposition erforderlich, oder es müssen über einen Zeitraum von mehreren Wochen zunächst Leerungs- und Füllstandsdaten zur präzisen Kalibrierung der Algorithmen manuell erhoben werden. Bei kurz getakteten Mess- und Übertragungsintervallen sind häufige Batteriewechsel der Fall, die in der Regel nur vom Sensoranbieter vorgenommen werden können. Herkömmliche Behälter verfügen in der Regel über keine Befestigungsmöglichkeiten für Sensoren, so dass eine Aufnahmevorrichtung selbst hergestellt werden muss. Als gering-invasiv hat sich die Nutzung von starken Industriemagneten bewährt. Beim großflächigeren Einsatz von Sensoren über kleinteilige Pilotprojekte hinaus ließen sich aufwandsseitig jedoch Skalen- und Synergieeffekte realisieren. Bei Sensorik, die außen auf dem Behälter aufgebracht wird, ist der Installationsaufwand deutlich geringer und es lassen sich Betriebskosten durch Energy-Harvesting-Ansätze reduzieren. Jedoch sind außen angebrachte Sensoren anfälliger für Diebstahl und Vandalismus. Die Nutzung einer eigenen LoRaWAN-Infrastruktur erscheint insbesondere aus Kostengründen im kommunalen Umfeld zunächst attraktiv, die Eignung hinsichtlich Abdeckung, Stabilität und Zuverlässigkeit ist pro Standort und Kommune jedoch immer individuell zu prüfen.

Entsorgungsunternehmen muss klar sein, dass es sich bei Abfall-Füllstandssensoren nicht um präzise Messwerkzeuge handelt, was sich in teilweise hohen Abweichungen der Messwerte zum realen Füllstand und signifikanten Standardabweichungen unter ähnlichen Füllgraden widerspiegelt. Es treten häufig Ausreißer und Anomalien, also etwa unerwartete Spitzen durch Positionierung von Abfällen direkt unter dem Sensor, in den Messwerten auf. Eine Arbeit mit Füllstanden, die grob in $25 \%$-Quartile unterteilt sind, erscheint technisch sinnvoll und ist hinreichend genau zur Umsetzung von Anwendungsfällen in der Entsorgungswirtschaft.

Als Erkenntnis der Untersuchung ist festzuhalten, dass die traditionellen Abfuhrintervalle insbesondere für grundstücksbezogene Behälter und Fraktionen wie Rest- 
müll, LVP und PPK in der Regel gut funktionieren, da nur selten eine zu frühe oder zu späte Abfuhr beobachtet werden konnte. Durch die nur schlecht vorhersagbare und durch äußere Einflüsse bedingte Befüllungsdynamik in öffentlichen Behältern bieten sich hier vermeintlich größere Potenziale für die Nutzung von Sensoren.

\subsection{Kritische Reflektion der Nutzenpotenziale}

In diesem Abschnitt diskutieren wir Herausforderungen für die Realisierung der zu Beginn des Beitrags skizzierten Nutzenpotenziale zur sensorbasierten Optimierung von Abfalllogistikprozessen vor dem Hintergrund der Erfahrungen aus den Pilotprojekten. Es muss zunächst die wirtschaftliche Belastung durch initiale Investitionen in Hardware und Software genannt werden, die insbesondere von kleineren Unternehmen und Kommunen nicht ohne Weiteres gestemmt werden können und derzeit auch nur schwierig als Teil der kommunalen Abfallgebühren abbildbar sind.

Grundlegende technische Herausforderungen sind die grundsätzliche Ungenauigkeit der Sensoren und fehlende Standards beim Einbau, der Konfiguration und den Schnittstellen. Die Ungenauigkeiten ließen sich prinzipiell durch kurze Messintervalle und Aufbereitung der Daten adressieren, was jedoch zu kurzen Batterielaufzeiten führt. Sensorik-Anbieter sind zudem oft daran interessiert, zusammen mit ihren Messtechnologien weitere proprietäre Softwareprodukte zu vertreiben und verkennen dabei oft, dass in der Entsorgungswirtschaft bereits weitestgehend Tourenplanungssysteme und auch IoT-Plattformen im Einsatz sind. Ziel von Entsorgungsunternehmen sollte im ersten Schritt sein, die durch Füllstandssensoren neu gewonnenen Informationen intelligent in vorhandene Systeme und Logistikprozesse zu integrieren, um schnell, unkompliziert und weniger kostenintensiv von den offenkundigen Vorteilen der Smart Bins zu profitieren.

Große Herausforderungen bestehen in der logistischen Integration der Sensordaten in die Unternehmenssysteme und Prozesse. Für eine füllstandsabhängige Disposition ist es einerseits zunächst erforderlich, dass die Rohdaten um Ausreißer und Anomalien bereinigt werden. Andererseits sind für die Disposition primär nicht die Echtzeit-Daten wesentlich, sondern hinreichend genaue Prognosen, wann ein Füllstand von beispielsweise $80 \%$ mit hoher Wahrscheinlichkeit erreicht wird, da die Disposition der Abfuhr in der Regel eine gewisse Vorlaufzeit benötigt und nicht erst am selben Tag stattfinden kann, wenn der Behälter bereits voll ist. Die Integration in die Tourenplanung ist so komplex, dass unklar ist, ob ein Lösungsalgorithmus das sogenannte Vehicle-Routing-Problem unter Einbeziehung von Sensordaten noch effizient und realitätsnah lösen kann, ohne dabei Nebenbedingungen (z. B. Fahrzeugund Ladekapazitäten, Zufahrtsbeschränkungen, Zeitfenster, Auslastung der Kolonnen) zu verletzen (Henke et al. 2015; Prasad und Kureethara 2018). Das vorliegende Problem ist daher NP-schwer, was bedeutet, dass es trotz zunehmender Fortschritte hinsichtlich Rechnerkapazitäten nicht mittels allgemeingültigen Lösungsansätzen in Polynomialzeit, das heißt in einem überschaubaren Zeitraum, gelöst werden kann (NP: nichtdeterministische Polynomialzeit). Durch diese praktische Unlösbarkeit beziehungsweise den exponentiell steigenden Aufwand für die Lösungssuche kommen für die Praxis nur heuristische Verfahren für die Suche nach einer Näherungslösung in Frage. Weiterhin haben Untersuchungen gezeigt, dass in Siedlungsstrukturen, 
in denen kleinere Behälter überwiegen (Ein- und Zweifamilienhausbebauung), von den Erzeugern bereits eine füllstandsabhängige Bereitstellung der Abfallbehälter erfolgt (Sabrowski 2016). Bei kleineren Behältern erscheint die Initiierung einer OnDemand-Leerung via Mail, App, Messenger-Anwendungen oder „Dash Buttons“ (Althoff et al. 2019) vielversprechender und kostengünstiger als der Einsatz von Sensorik. Auch „Mängelmelder-Apps“ für BürgerInnen in Verbindung mit QR-Codes insbesondere an öffentlichen Behältern können eine effektive und günstigere Lösung zur Steuerung der Disposition als Anschaffung und Betrieb von Sensorik darstellen (Stadtreinigung Hamburg 2018). Doch die Integration der Daten ist nur ein Teil des Problems, meist fehlt es in Entsorgungsunternehmen zudem an einer eindeutigen Vision, wie die digitale Transformation für das Geschäftsmodell vonstattengehen soll (Schlaepfer und Welz 2017). Strukturen und Prozesse in der Entsorgungswirtschaft weisen eine geringe „Digital Readiness“ auf (Wilts und Berg 2018) und es bedarf enormer Überzeugungsarbeit bei Entscheidungsträgern, um ein langfristiges KostenNutzen-Kalkül zu platzieren. Gerade die fehlenden praktischen Vorbilder für komplexe Standortplanungs-, Touren- und Optimierungsprobleme stellen ein Hindernis bei der Umsetzung dar.

Die Technologien, die benötigt werden, um Abfallbehälter, Kunden und Entsorgungsunternehmen in Echtzeit zu verbinden existieren bereits heute (Hawlitschek 2021). Ob ein entsprechendes System in Summe aber effektiver und kostengünstiger funktioniert als die starren, auf Erfahrungswerten basierten ,Milkrun“-Verfahren ist nach Ansicht der Autoren noch nicht abschließend geklärt. Als alternativen Anwendungsfall, abseits der oft diskutierten Dynamisierung von Abfalllogistikprozessen, ließen sich Füllstandssensoren etwa in wechselnden Behältern verwenden, um turnusmäßig die Abfuhrintervalle und Angemessenheit der Behältervolumen zu prüfen und zu optimieren. Der Einsatz von Sensoren in diesen Anwendungsfall kann die Notwendigkeit von Vor-Ort-Sichtprüfungen von Abfallkontrolleuren teilweise reduzieren oder die gleichzeitige Überprüfung einer größeren Anzahl an Behältern bei gleichem Personaleinsatz ermöglichen.

\subsection{Entwicklungswege zur Hebung der Nutzenpotenziale}

Zur Adressierung der zuvor identifizierten Herausforderungen zur Realisierung intelligenter Abfalllogistikprozesse erwarten wir zunächst von Sensorherstellern eine Ausweitung des Angebots von umfassenden Betriebs- und Servicepaketen, etwa über die Realisierung von As-a-Service-Modellen. Zusätzlich sind vereinfachte Verfahrensweisen sowohl für die Remote-Konfiguration und Statusbenachrichtigungen als auch das Testen und Kalibrieren von Sensoren vor Ort wünschenswert. Weiterhin sehen die Autoren im Bereich der Konnektivität das größte Potenzial in LPWANTechnologien. Hier erscheinen Varianten mit NB-IoT/LTE-M oder LoRaWAN, gegebenenfalls mit einem Doppel-Modem zur Erhöhung der Einsatzflexibilität, aufgrund der Übertragungsraten und Energieeffizienz am sinnvollsten. Um gleichzeitig Energie zu sparen und Fehlalarme zu vermeiden, kann eine wie von Kamm et al. (2020) beschriebene „Selbstabgleichfunktion“ hilfreich sein, die anhand von historischer Fülldaten direkt auf dem Sensor das Füllverhalten analysiert, Anomalien erkennt und selbstständig entscheidet, wann Datenmessungen und -übertragungen sinnvoll 
sind. Bei bisher verfügbaren Sensoren beschränken sich die Fähigkeiten bislang weitestgehend auf die Berechnung von Mittelwerten über einen geringen Zeitraum. Zusätzlich sollten sich Sensoranbieter auf die Bereitstellung und Absicherung von gut dokumentierten Schnittstellen und weniger auf die Entwicklung umfangreicher Tourenplanungssysteme oder IoT-Silos fokussieren. Behälterhersteller können zur Entwicklung beitragen, indem sie standardisierte Einbauvorrichtungen für Sensoren vorsehen und notwendige Parameter (z.B. Innenhöhen) zur Kalibrierung in einem genormten Format bereitstellen.

Als offenes Forschungsfeld für Wissenschaft und Praxis stellt sich die Entwicklung intelligenter Algorithmen zur Bereinigung, Berechnung und Prognose von Füllständen dar, die neben historischen Messwerten idealerweise auch andere Datenquellen wie Umwelt- und Bewegungsdaten oder Ferienkalender berücksichtigen, um trotz saisonalen Schwankungen und unvorhergesehenen Ereignissen hinreichend genaue Ergebnisse zu liefern. Weiterführend steht auch die Entwicklung einer ITReferenzarchitektur für Smart Bins in Bezug auf Gerätekonnektivität, Datenmodellierung, Datenanalyse, Visualisierung, Konnektoren und Sicherheit noch aus (Lempert und Pflaum 2019). Bisher sind in der Anbieterlandschaft keine einheitlichen Datenstrukturen und -modelle zu beobachten, was eine Integration in die bestehende Systemlandschaft erschwert. Um die Interoperabilität verschiedener Sensoren und Systeme zu vereinfachen, bietet sich hierbei die Nutzung eines einheitlichen und offenen Standards wie NGSI-LD, ein internationaler Standard für das Kontextinformationsmanagement im Bereich Smart Cities, an (ETSI 2019; FIWARE 2020). Insbesondere die Generierung von Prognoseinformationen und die Integration mit bestehenden Tourenplanungs- und Betriebsführungssysteme stellt derzeit die größte Herausforderung dar, bei der Forschende aus dem Bereich der Logistik als auch ITUnternehmen aufgefordert sind, intelligente und übertragbare Lösungen zu entwickeln.

Kommunen und die Entsorgungswirtschaft schlussendlich, sollten den Aufbau einer föderierten Datenbasis in Erwägung ziehen, da Prognosealgorithmen umso bessere Ergebnisse erzielen, je mehr Trainingsdaten zur Verfügung stehen. Dabei sollte auch ein intensiver Erfahrungsaustausch von großen Entsorgungsunternehmen mit kleineren Unternehmen aktiv vorangetrieben werden. Regulatorisch müssen dynamisierte Leerungsszenarien in den kommunalen Abfallgebührensatzungen abgebildet werden. In diesem Rahmen werden auch Diskussionen zu führen sein, inwiefern eine finanzielle Beteiligung der (privatwirtschaftlich organisierten) Dualen Systeme für Leichtverpackungen bei der Ausrüstung von Wertstoffbehältern mit Sensorik möglich ist. Maßgeblich müssen sich Entsorgungsunternehmen zunächst mit einer übergeordneten Digitalisierungsstrategie und -vision sowie den dafür benötigten Kompetenzen und Ressourcen auseinandersetzen, bevor sensorbasierte Optimierungsszenarien sinnvoll umgesetzt werden können.

\section{Fazit}

Die zunehmende Digitalisierung und der Wandel der Gesellschaft erfordern auch neue Ansätze für eine intelligente und nachhaltige Entsorgungswirtschaft. Vor die- 
sem Hintergrund stellte dieser Beitrag das Konzept der Smart Bins und mögliche Nutzenpotenziale dar. Der Fokus der Untersuchung lag dabei insbesondere auf der sensorbasierten Optimierung von Abfalllogistikprozessen. Im Rahmen einer multiplen Fallstudie mit mehreren Entsorgungsunternehmen wurde der Einsatz von Füllstandssensoren evaluiert. Anhand der praktischen Erkenntnisse wurden Herausforderungen identifiziert, die bei einem großflächigen Einsatz von Sensoren in Abfallbehältern zur Dynamisierung der Abfalllogistik adressiert werden müssen. Die Studie hat gezeigt, dass die Integration von Sensorik in die Prozesse der Entsorgungslogistik in Zukunft Veränderungen in der Arbeitsorganisation und den betrieblichen Abläufen mit sich bringen wird und noch weitreichenden Entwicklungsbedarf erfordert.

Abschließend ist festzuhalten, dass diese Untersuchung lediglich einen ersten Schritt darstellt, um sich dem komplexen Thema zu nähern. Auch wenn die Erkenntnisse auf dem praktischen Einsatz in Pilotprojekten bei drei großen Entsorgungsunternehmen basieren, konnte die Technologie nur über einen begrenzten Zeitraum und nicht abschließend in allen Behältern und mit allen Fraktionen erprobt werden. Zudem werden weitere anwendungsorientierte Untersuchungen benötigt, welche praktikable Lösungen für die identifizierten Herausforderungen entwickeln.

Danksagung An dieser Stelle möchten wir uns bei unseren Kolleginnen und Kollegen Stefan Andreef, Sarah Bonnemann, Gereon Duesmann, Volker Reck, Nathalie Schilly und Jochen Schmitz für ihr engagiertes Mitwirken im Rahmen der Projekte bedanken. Unser Dank geht auch an die einzelnen Umsetzpartner, welche die Projekte durch ihr Know-How und ihre Unterstützung ermöglicht haben.

Funding Open Access funding enabled and organized by Projekt DEAL.

Open Access Dieser Artikel wird unter der Creative Commons Namensnennung 4.0 International Lizenz veröffentlicht, welche die Nutzung, Vervielfältigung, Bearbeitung, Verbreitung und Wiedergabe in jeglichem Medium und Format erlaubt, sofern Sie den/die ursprünglichen Autor(en) und die Quelle ordnungsgemäß nennen, einen Link zur Creative Commons Lizenz beifügen und angeben, ob Änderungen vorgenommen wurden.

Die in diesem Artikel enthaltenen Bilder und sonstiges Drittmaterial unterliegen ebenfalls der genannten Creative Commons Lizenz, sofern sich aus der Abbildungslegende nichts anderes ergibt. Sofern das betreffende Material nicht unter der genannten Creative Commons Lizenz steht und die betreffende Handlung nicht nach gesetzlichen Vorschriften erlaubt ist, ist für die oben aufgeführten Weiterverwendungen des Materials die Einwilligung des jeweiligen Rechteinhabers einzuholen.

Weitere Details zur Lizenz entnehmen Sie bitte der Lizenzinformation auf http://creativecommons.org/ licenses/by/4.0/deed.de.

\section{Literatur}

Althoff T, Holzhauer R, Babnik E et al (2019) Effizienzsteigerung der Sammelsysteme für die bürgernahe Abfallerfassung. Müll Abfall. https://doi.org/10.37307/j.1863-9763.2019.04.07

Castro Lundin A, Ozkil AG, Schuldt-Jensen J (2017) Smart cities: a case study in waste monitoring and management. In: Proceedings of the 50th Hawaii International Conference on System Sciences

Destatis (2018) Beschäftigte, Umsatz, Produktionswert und Wertschöpfung der Unternehmen in der Energie- und Wasserversorgung: Deutschland, Jahre, Wirtschaftszweige

D’Onofrio S, Portmann E (2017) Cognitive computing in smart cities. Informatik Spektrum 40:46-57

Eisenhardt KM (1989) Building theories from case study research. Acad Manage Rev 14:532-550

ETSI (2019) Context Information Management (CIM). NGSI-LD API 
FIWARE (2020) Fiware Data Models. In: FIWARE. https://www.fiware.org/developers/data-models/. Zugegriffen: 16. Jan. 2021

Giffinger R, Fertner C, Kramar H et al (2007) Smart Cities-Ranking of European medium-sized cities. Vienna University of Technology, Wien

Hawlitschek F (2021) Interview with Benjamin Scheffler on The Future of Waste Management. Bus Inf Syst Eng 63:207-211

Hawlitschek F, Teubner T, Gimpel H (2018) Consumer motives for peer-to-peer sharing. J Clean Prod 204:144-157

Heller M, Langheim J (2018) Analyse des Münchner 3-Tonnen-Systems für den Abfallwirtschaftsbetrieb München. Münchner Statistik, 2. Quartalsheft, Jahrgang 2018:24-33

Henke T, Speranza MG, Wäscher G (2015) The multi-compartment vehicle routing problem with flexible compartment sizes. Eur J Oper Res 246:730-743

Hoffmann D (2018) Shaping wellsprings of innovation: towards organizational design configurations for digital innovation management. In: Proceedings of the 26th European Conference on Information Systems (ECIS) Portsmouth

Höfle KA, Althoff T, Holzhauer R (2019) Automatisierung der optischen Bewertung von Siedlungsabfällen mittels künstlicher Intelligenz. Müll Abfall. https://doi.org/10.37307/j.1863-9763.2019.03.06

Kamm M, Gau M, Schneider J, Vom Brocke J (2020) Smart waste collection processes-A case study about smart device implementation. In: Proceedings of the 53rd Hawaii International Conference on System Sciences

Kang Y, Zhao Z, Magdy A et al (2019) Scalable multi-resolution spatial visualization for anthropogenic litter data. In: Proceedings of the 27th ACM SIGSPATIAL International Conference on Advances in Geographic Information Systems, S 560-563

Kranert M (Hrsg) (2017) Einführung in die Kreislaufwirtschaft, 5. Aufl. Springer Vieweg, Wiesbaden

Lempert S, Pflaum A (2019) Vergleichbarkeit der Funktionalität von IoT-Software-Plattformen durch deren einheitliche Beschreibung in Form einer Taxonomie und Referenzarchitektur. HMD 56:1178-1203

McDonough W, Braungart M (2003) Cradle to cradle: remaking the way we make things, 1. Aufl. Macmillan, New York

Mittermayr R, Klünsner S (2020) Smart Waste. In: Sihn-Weber A, Fischler F (Hrsg) CSR und Klimawandel: Unternehmenspotenziale und Chancen einer nachhaltigen und klimaschonenden Wirtschaftstransformation. Springer, Berlin, Heidelberg, S 425-437

Prasad S, Kureethara JV (2018) Optimizing milk run and use of bin-packing in waste collection problems. Int J Eng Technol 7:577-579

Sabrowski R (2016) Hausmüllanalyse der Stadt Jena: Endbericht 2015/2016, Jena

Santjer M (2018) Ermittlung der Raumdichten von Rest- und Bioabfall. Institut für Abfall, Abwasser und Infrastruktur-Management $\mathrm{GmbH}$, Ahlen

Schlaepfer K, Welz M (2017) Das dynamische Unternehmen: Wie Wertewandel, Innovation und Digitalisierung zum Erfolg führen. Schäffer-Poeschel, Stuttgart

Sein MK, Henfridsson O, Purao S et al (2011) Action design research. MISQ 35:37-56

Song Q, Li J, Zeng X (2015) Minimizing the increasing solid waste through zero waste strategy. J Clean Prod 104:199-210

Stadt Halle (2014) Endbericht: Durchführung einer Hausmüllanalyse in der Stadt Halle (Saale). Stadt Halle, Halle

Stadtreinigung Hamburg (2018) kehrseite 1/2018. In: Stadtreinigung Hamburg. https://www.stadtreinigung. hamburg/wohnungswirtschaft/downloads/. Zugegriffen: 16. Jan. 2021

United Nations (2015) Transforming Our World: the 2030 Agenda for Sustainable Development. United National General Assembly, New York

VKU (2020) Unterflursysteme II - Eine innovative Art der Hausmüllerfassung. Verband kommunaler Unternehmen e. V., Berlin

Wilts $\mathrm{CH}$, Berg H (2018) The digital circular economy: can the digital transformation pave the way for resource-efficient materials cycles? Int J Environ Sci Nat Resour 7:1-4

Yerraboina S, Kumar NM, Parimala KS, Jyothi NA (2018) Monitoring the smart garbage bin filling status: an IoT application towards waste management. Int J Civ Eng Technol 9:373-381

Yoo Y, Boland RJ, Lyytinen K, Majchrzak A (2012) Organizing for innovation in the digitized world. Organ Sci 23:1398-1408

Zaman AU (2015) A comprehensive review of the development of zero waste management: lessons learned and guidelines. J Clean Prod 91:12-25 